\title{
In Situ Hybridization and Reverse Transcription-Polymerase Chain Reaction for Cyclin D1 mRNA in the Diagnosis of Mantle Cell Lymphoma in Paraffin-Embedded Tissues
}

Evangelia Athanasiou, M.D., Vassiliki Kotoula, M.D., Prodromos Hytiroglou, M.D., Sophia Kouidou, Ph.D., Vassiliki Kaloutsi, M.D., Constantine S. Papadimitriou, M.D. Departments of Pathology (EA, VK, PH, VK, CSP) and Biochemistry (SK), Medical School of the Aristotle University, Thessaloniki, Greece

Mantle cell lymphoma (MCL) is characterized by the chromosomal translocation $\mathrm{t}(11 ; 14)$, which involves rearrangement of the $b c l-1$ proto-oncogene to the immunoglobulin heavy chain gene and results in overexpression of cyclin D1 mRNA. In this study, we evaluated the diagnostic relevance of three methods that may be helpful in the diagnosis of MCL: in situ hybridization (ISH) and a stringent reverse transcriptase-polymerase chain reaction (RT-PCR) protocol for cyclin D1 mRNA, and immunohistochemistry for cyclin D1 protein. The study group included 37 paraffin-embedded specimens (25 from lymph nodes and 12 from extranodal tissues) from 30 patients. MCL diagnosis was performed according to the Revised European-American Classification of Lymphoid Neoplasms. Twenty-nine patients with non-MCL lymphoproliferative disorders comprised the control group. Biotin-labeled ISH was performed in 28 cases of MCL, $24(86 \%)$ of which were found to be positive. As shown by ISH in extranodal tissues, cyclin D1 mRNA was present not only in neoplastic lymphoid cells, but in other cell types as well. For this reason, RT-PCR results were considered reliable for MCL diagnosis only on informative material (from tissues that do not normally express cyclin D1); this method was evaluated as positive in 16 of 18 (89\%) MCL cases. Cyclin D1 immunopositivity was present in 20 of $29(69 \%)$ MCL cases. No members of the control group were found to express cyclin D1 mRNA by either ISH or RT-PCR under the stringent conditions used. In conclusion,

Copyright (C) 2001 by The United States and Canadian Academy of Pathology, Inc.

VOL. 14, NO. 2, P. 62, 2001 Printed in the U.S.A.

Date of acceptance: November 15, 2000.

This work was supported in part by the Research Committee of the Aristotle University of Thessaloniki (code 4902).

Address reprint requests to: Constantine S. Papadimitriou, M.D., Department of Pathology, Aristotle University Medical School, 54006 Thessaloniki, Greece; fax: (011-3031) 999229. stringent RT-PCR for cyclin D1 expression can be helpful in MCL diagnosis in paraffin-embedded material from lymph nodes. ISH is a sensitive method for cyclin D1 mRNA detection; its sensitivity is superior to that of cyclin D1 immunohistochemistry and similar to that of the stringent RT-PCR used. ISH is very specific as well, clearly more specific than RT-PCR, because it allows the correlation of molecular findings with morphology. This method can be applied on all types of paraffin-embedded tissues and provides an accurate tool for MCL diagnosis.

KEY WORDS: Cyclin D1, In situ hybridization, Mantle cell lymphoma, RT-PCR.

Mod Pathol 2001;14(2):62-71

Mantle cell lymphoma (MCL), previously known as centrocytic lymphoma, has been recognized as a distinct clinicopathologic entity since 1982 (1) and represents 3 to $9 \%$ of all non-Hodgkin lymphomas in Western countries (2). In addition to affecting the lymph nodes, this neoplasm may affect the gastrointestinal tract, spleen, bone marrow, and, less frequently, the pharynx and the skin. MCL cells in peripheral blood may mimic chronic lymphocytic leukemia (3). Because MCL has a more aggressive course than low-grade lymphomas of B-cell origin, accurate diagnosis of this neoplasm is of great clinical importance $(4,5)$.

When histologic sections are examined, MCL is found to exhibit a relatively monotonous neoplastic cell population, composed either of medium-sized lymphoid cells with cleaved nuclei (centrocytoid type), or of larger cells with more cytoplasm, round to oval nuclei with coarsely dispersed chromatin, and a high mitotic rate (blastoid type). The histologic differential diagnosis of MCL of centrocytoid type includes several low-grade lymphoproliferative disorders, such as B-cell chronic lymphocytic 
leukemia (B-CLL), marginal zone lymphoma (MZL), and MALT-type lymphoma, whereas in cases of the blastoid type, the possibility of lymphoblastic lymphoma must be ruled out. Specific morphologic, immunohistochemical, and molecular markers for the accurate diagnosis of MCL have been outlined in the Revised European-American Classification of Lymphoid Neoplasms (6) and have been included in the recent lymphoma classification of the World Health Organization (7).

The chromosomal translocation $\mathrm{t}(11 ; 14)(\mathrm{q} 13$; q32), which is the characteristic molecular alteration of MCL, brings the bcl-1 (PRAD-1) gene close to the immunoglobulin heavy chain gene on chromosome 14 and results in overexpression of cyclin D1, a cell cycle regulator.

This translocation has been identified in 60 to $70 \%$ of MCL cases by karyotypic analysis or Southern blot (8-11) and in 95\% of cases by fluorescence in situ hybridization (ISH) performed on frozen tissues or intact cells (12). However, the sensitivity of these methods in paraffin-embedded material is very low; instead, polymerase chain reaction (PCR)based methods (13) are more sensitive. The incidence of $\mathrm{t}(11 ; 14)$ in neoplastic lymphoid disorders other than MCL is fairly low $(14,15)$.

By a reverse transcriptase-PCR (RT-PCR) assay on paraffin-embedded material, Ives Aguilera et al. (16) have recently demonstrated the presence of cyclin D1 mRNA in 95\% of MCL, but also in a high percentage of various other lymphoproliferative disorders, such as MZL, hairy cell leukemia, multiple myeloma, and prolymphocytic leukemia (16, 17). Similar rates of cyclin D1 expression were found by competitive RT-PCR in frozen tissues of MCL and other B-cell lymphomas (18), verifying the results of earlier studies utilizing Northern blot (19) and ISH (20), but questioning the specificity of RTPCR for MCL diagnosis.

The aim of our study was to evaluate the usefulness of the presently available molecular methods for MCL diagnosis in everyday pathology practice. We used paraffin-embedded material for ISH and RT-PCR for cyclin D1 mRNA; we also performed immunohistochemistry for cyclin D1, as well as nested PCR for the detection of $t(11 ; 14)$.

\section{MATERIALS AND METHODS}

\section{Tissue Specimens}

The consultation files of our department were searched for cases of MCL. Thirty-seven paraffinembedded specimens from 30 patients were found and used in the present study. These included 24 samples from lymph nodes, 3 bone marrow biopsies, and 10 extranodal tissues (spleen, $n=1$; stomach, $n=3$; small intestine, $n=1$; liver, $n=1$; skin, $n=1$; palate, $n=1$; nasopharynx, $n=2$ ) (Table 1). MCL diagnosis was based on the criteria of the Revised European-American Classification of Lymphoid Neoplasms. At histologic examination, the tissues were infiltrated, either focally or diffusely, by a monotonous neoplastic population of mediumsized lymphoid cells with cleaved nuclei (Fig. 1A, B). Twenty cases were identified as MCL of centrocytoid type and 10 as MCL of blastoid type. The immunohistochemical profile of the neoplastic cells included positive staining for the antigens CD20 and CD45RA, and negative staining for CD3, CD45RO, CD23, CD34, and TdT. CD5 antigen was also included in the immunohistochemistry (IHC) panel, because this antigen is known to be expressed in the majority of cases of MCL.

In addition, a control group of 29 selected cases of non-Hodgkin lymphoma was created for the purpose of determining the specificity of the findings. This group included 10 cases of B-CLL (lymph node, $n=7$; bone marrow, $n=3$ ), 5 cases of MZL (lymph node, $n=1$; spleen, $n=2$; bone marrow, $n$

TABLe 1. Mantle Cell Lymphoma Patient Cases, Specimens, and Primary Diagnosis

\begin{tabular}{|c|c|c|c|c|c|c|}
\hline $\begin{array}{l}\text { Patient } \\
\text { No. }\end{array}$ & $\begin{array}{l}\text { Tissue } \\
\text { Specimen }\end{array}$ & $\begin{array}{l}\text { Histologic } \\
\text { Type }\end{array}$ & CD23 & CD34 & $\mathrm{TdT}$ & CD5 \\
\hline 1 & $\mathrm{LN}$ & $\mathrm{CV}$ & $\mathrm{N}$ & & & $P$ \\
\hline 2 & LN & $\mathrm{CV}$ & $\mathrm{N}$ & & & $\mathrm{P}$ \\
\hline 3 & LN & $\mathrm{CV}$ & $\mathrm{N}$ & & & $\mathrm{P}$ \\
\hline 4 & LN & $\mathrm{CV}$ & $\mathrm{N}$ & & & $\mathrm{P}$ \\
\hline 5 & LN & $\mathrm{CV}$ & $\mathrm{N}$ & & & $\mathrm{P}$ \\
\hline 6 & LN & $\mathrm{CV}$ & $\mathrm{N}$ & & & $\mathrm{P}$ \\
\hline 7 & $\mathrm{LN}$ & $\mathrm{CV}$ & $\mathrm{N}$ & & & $\mathrm{P}$ \\
\hline 8 & LN & $\mathrm{CV}$ & $\mathrm{N}$ & & & $\mathrm{P}$ \\
\hline 9 & LN & $\mathrm{CV}$ & $\mathrm{N}$ & & & $\mathrm{P}$ \\
\hline 10 & LN & $\mathrm{CV}$ & $\mathrm{N}$ & & & $\mathrm{P}$ \\
\hline 11 & LN & $\mathrm{CV}$ & $\mathrm{N}$ & & & $\mathrm{P}$ \\
\hline 12 & LN & $\mathrm{CV}$ & $\mathrm{N}$ & & & $\mathrm{P}$ \\
\hline 13 & $\mathrm{LN}$ & CV & $\mathrm{N}$ & & & $\mathrm{P}$ \\
\hline 14 & LN & $\mathrm{CV}$ & $\mathrm{N}$ & & & $\mathrm{N}$ \\
\hline 15 & Palate $^{a}$ & $\mathrm{CV}$ & $\mathrm{N}$ & & & $\mathrm{N}$ \\
\hline 16 & LN & BV & $\mathrm{N}$ & & & $\mathrm{P}$ \\
\hline 17 & LN & BV & $\mathrm{N}$ & $\mathrm{N}$ & $\mathrm{N}$ & $\mathrm{P}$ \\
\hline 18 & LN & BV & $\mathrm{N}$ & $\mathrm{N}$ & $\mathrm{N}$ & $\mathrm{N}$ \\
\hline 19 & LN & BV & $\mathrm{N}$ & $\mathrm{N}$ & $\mathrm{N}$ & $\mathrm{P}$ \\
\hline 20 & LN & BV & $\mathrm{N}$ & $\mathrm{N}$ & $\mathrm{N}$ & $\mathrm{P}$ \\
\hline 21 & LN & BV & $\mathrm{N}$ & $\mathrm{N}$ & $\mathrm{N}$ & $\mathrm{P}$ \\
\hline 22 & LN & BV & $\mathrm{N}$ & $\mathrm{N}$ & $\mathrm{N}$ & $\mathrm{P}$ \\
\hline 23 & $\mathrm{LN}$ & BV & $\mathrm{N}$ & $\mathrm{N}$ & $\mathrm{N}$ & $\mathrm{P}$ \\
\hline 24 & Nasopharynx $^{a}$ & BV & $\mathrm{N}$ & $\mathrm{N}$ & $\mathrm{N}$ & $P$ \\
\hline 25 & Skin & BV & $\mathrm{N}$ & $\mathrm{N}$ & $\mathrm{N}$ & $\mathrm{P}$ \\
\hline 26 & BMB & $\mathrm{CV}$ & $\mathrm{N}$ & & & $\mathrm{P}$ \\
\hline 26 & Small intestine & $\mathrm{CV}$ & $\mathrm{N}$ & & & $\mathrm{P}$ \\
\hline 26 & Stomach & $\mathrm{CV}$ & $\mathrm{N}$ & & & $\mathrm{N}$ \\
\hline 27 & LN & $\mathrm{CV}$ & $\mathrm{N}$ & & & $\mathrm{N}$ \\
\hline 27 & Liver & $\mathrm{CV}$ & $\mathrm{N}$ & & & $\mathrm{P}$ \\
\hline 27 & Spleen & $\mathrm{CV}$ & $\mathrm{N}$ & & & $\mathrm{N}$ \\
\hline 28 & Stomach & $\mathrm{CV}$ & $\mathrm{N}$ & & & $\mathrm{N}$ \\
\hline 28 & BMB & $\mathrm{CV}$ & $\mathrm{N}$ & & & $\mathrm{N}$ \\
\hline 29 & LN & $\mathrm{CV}$ & $\mathrm{N}$ & & & $\mathrm{P}$ \\
\hline 29 & BMB & CV & $\mathrm{N}$ & & & $\mathrm{N}$ \\
\hline 30 & Stomach & CV & $\mathrm{N}$ & & & $\mathrm{P}$ \\
\hline 30 & LN & CV & $\mathrm{N}$ & & & P \\
\hline
\end{tabular}

$\mathrm{BMB}$, bone marrow biopsy; $\mathrm{BV}$, blastoid variant; $\mathrm{CV}$, centrocytoid variant; LN, lymph node; $\mathrm{N}$, negative; $\mathrm{P}$, positive.

${ }^{a}$ Without epithelium. 

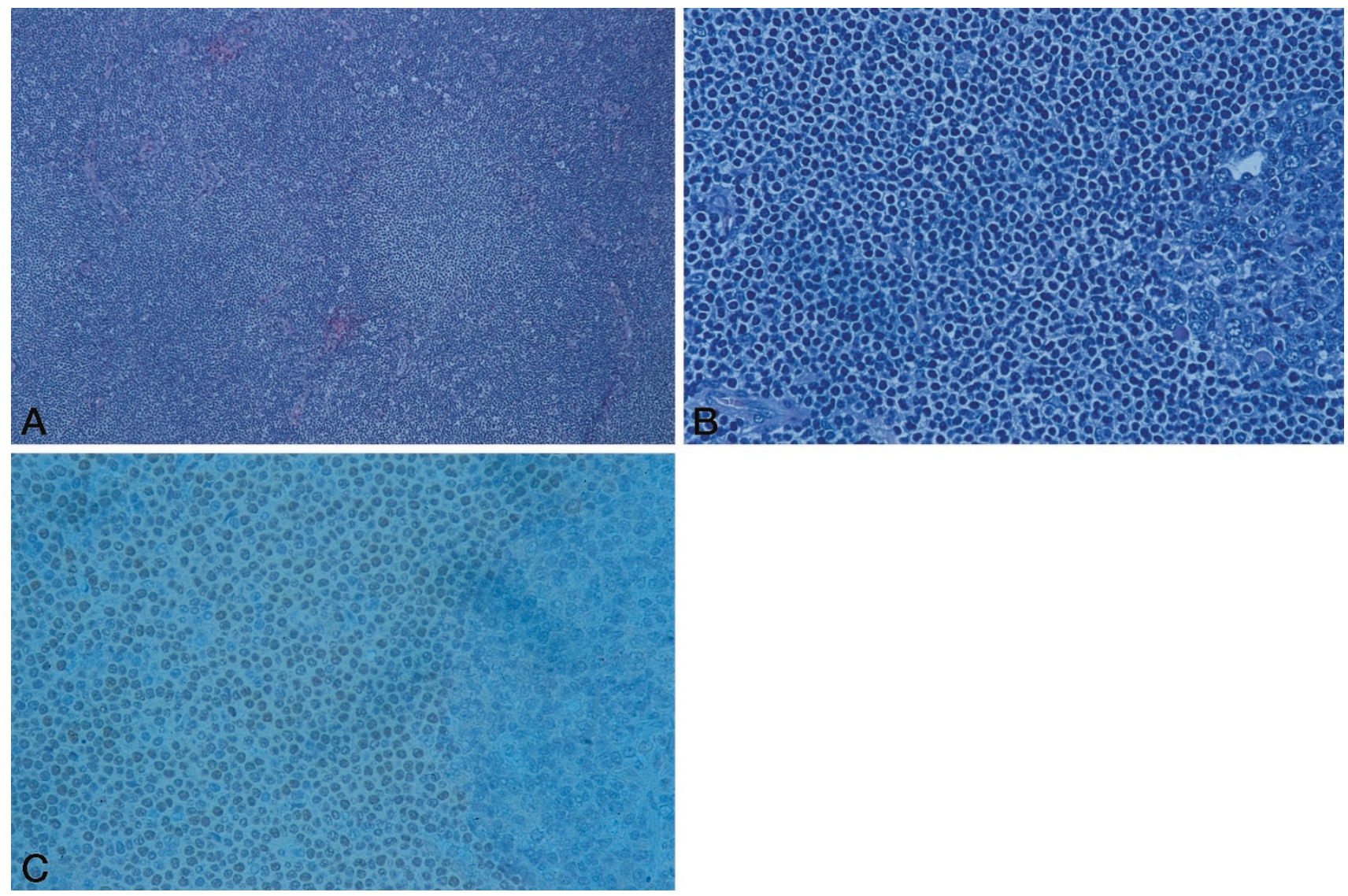

FIGURE 1. Histologic and immunohistochemical findings. A, low-power view of a lymph node infiltrated by mantle cell lymphoma (MCL), centrocytoid type. A vaguely nodular pattern can be seen. Original magnification, $100 \times$. B, high-power view showing a monotonous population of MCL cells. Original magnification, $400 \times$. C, positive nuclear immunostaining for cyclin D1 is present in the neoplastic lymphoid cells, whereas the germinal center cells are negative. Original magnification, $400 \times$.

= 2), 4 cases of MALT-type lymphoma (lymph node, $n=1$; gastric biopsy, $n=2$; bone marrow, $n$ $=1$ ), 4 cases of lymphoblastic lymphoma with lymph node involvement, and 3 bone marrow biopsies with hairy cell leukemia (Table 2). These cases were selected because they represent disorders that are often difficult to differentiate from MCL on the basis of histologic and immunohistochemical features. In particular, the cases of hairy cell leukemia were included because of the occasional positivity of this disorder for cyclin D1 protein (21). Three normal bone marrow specimens were included in the control group as well.

\section{ISH for Cyclin D1 mRNA}

Four-micron-thick paraffin sections were placed on positively charged slides and incubated overnight at $57^{\circ} \mathrm{C}$. For hybridization, the Super Sensitive mRNA Detection System (Biogenex, San Ramon, CA) and an oligonucleotide cyclin D1, biotinlabeled probe (Biogenex) were used. Minor modifications to the manufacturer's protocol were applied, such as shortening of the proteolysis incubation time (15 min) and increasing the posthybridization washing temperature $\left(54^{\circ} \mathrm{C}\right)$. The ad- dition of levamizole was necessary to quench the endogenous alkaline phosphatase activity, which is expressed at high level in bone marrow neutrophils. The chromogen used was BCIP/NBT (Biogenex). "Poly-A" (Biogenex) was used as a positive control for the demonstration of mRNA on parallel sections. Sections with gastrointestinal epithelium, which normally expresses cyclin D1, were used as a positive control.

\section{RNA Extraction and RT-PCR for Cyclin D1 mRNA}

Eight- to $10-\mu \mathrm{m}$-thick paraffin sections were incubated overnight in a digestion buffer $(10 \mathrm{~mm}$ $\mathrm{NaCl}, 50 \mathrm{~mm}$ Tris-HCl, pH 7.4, $20 \mathrm{~mm}$ ethylene diamine tetra-acetic acid, and $1 \%$ sodium dodecyl sulfate) at $55^{\circ} \mathrm{C}$. RNA was extracted from the completely digested tissue by use of TRIZOL-LS (Gibco), according to the manufacturer's instructions; where needed, 0.5 microl glycogen (BoehringerMannheim, Indianapolis, IN) were added as an RNA carrier during the first step of the method. For first-strand synthesis, half of the extracted RNA was labeled with random hexamers at $70^{\circ} \mathrm{C}$ for $10 \mathrm{~min}$ utes. Reverse transcription was performed with Superscript II (Gibco) at $42^{\circ} \mathrm{C}$ for 50 minutes, followed 
TABLE 2. Control Group Specimens, Histologic and Immunohistochemical Diagnosis ${ }^{a}$

\begin{tabular}{|c|c|c|c|c|c|c|}
\hline $\begin{array}{c}\text { Patient } \\
\text { No. }\end{array}$ & Diagnosis & Tissue & CD23 & CD34 & $\mathrm{TdT}$ & CD5 \\
\hline 1 & B-CLL & $\mathrm{LN}$ & $\mathrm{P}$ & $\mathrm{N}$ & $\mathrm{N}$ & $\mathrm{P}$ \\
\hline 2 & B-CLL & $\mathrm{LN}$ & $\mathrm{P}$ & $\mathrm{N}$ & $\mathrm{N}$ & $\mathrm{P}$ \\
\hline 3 & B-CLL & $\mathrm{LN}$ & $\mathrm{P}$ & $\mathrm{N}$ & $\mathrm{N}$ & $\mathrm{P}$ \\
\hline 4 & B-CLL & LN & $\mathrm{P}$ & $\mathrm{N}$ & $\mathrm{N}$ & $\mathrm{P}$ \\
\hline 5 & B-CLL & $\mathrm{LN}$ & $\mathrm{P}$ & $\mathrm{N}$ & $\mathrm{N}$ & $\mathrm{P}$ \\
\hline 6 & B-CLL & $\mathrm{LN}$ & $\mathrm{P}$ & $\mathrm{N}$ & $\mathrm{N}$ & $\mathrm{P}$ \\
\hline 7 & B-CLL & LN & $\mathrm{P}$ & $\mathrm{N}$ & $\mathrm{N}$ & $\mathrm{P}$ \\
\hline 8 & MZL & $\mathrm{LN}$ & $\mathrm{P}$ & $\mathrm{N}$ & $\mathrm{N}$ & $\mathrm{N}$ \\
\hline 9 & MZL & Spleen & $\mathrm{N}$ & $\mathrm{N}$ & $\mathrm{N}$ & $\mathrm{N}$ \\
\hline 10 & MZL & Spleen & $\mathrm{P} / \mathrm{N}$ & $\mathrm{N}$ & $\mathrm{N}$ & $\mathrm{N}$ \\
\hline 11 & MALT & $\mathrm{LN}$ & $\mathrm{N}$ & $\mathrm{N}$ & $\mathrm{N}$ & $\mathrm{N}$ \\
\hline 12 & MALT & Gastric & $\mathrm{N}$ & $\mathrm{N}$ & $\mathrm{N}$ & $\mathrm{N}$ \\
\hline 13 & MALT & Gastric & $\mathrm{P} / \mathrm{N}$ & $\mathrm{N}$ & $\mathrm{N}$ & $\mathrm{N}$ \\
\hline 14 & LBL & $\mathrm{LN}$ & $\mathrm{N}$ & $\mathrm{P}$ & $\mathrm{N}$ & $\mathrm{N}$ \\
\hline 15 & LBL & $\mathrm{LN}$ & $\mathrm{N}$ & $\mathrm{P}$ & $\mathrm{P}$ & $\mathrm{N}$ \\
\hline 16 & LBL & $\mathrm{LN}$ & $\mathrm{N}$ & $\mathrm{P}$ & $\mathrm{N}$ & $\mathrm{N}$ \\
\hline 17 & LBL & $\mathrm{LN}$ & $\mathrm{N}$ & $\mathrm{P}$ & $\mathrm{P}$ & $\mathrm{N}$ \\
\hline 18 & B-CLL & $\mathrm{BMB}$ & $\mathrm{P} / \mathrm{N}$ & $\mathrm{N}$ & $\mathrm{N}$ & $\mathrm{P} / \mathrm{N}$ \\
\hline 19 & B-CLL & $\mathrm{BMB}$ & $\mathrm{P} / \mathrm{N}$ & $\mathrm{N}$ & $\mathrm{N}$ & $\mathrm{P}$ \\
\hline 20 & B-CLL & $\mathrm{BMB}$ & $\mathrm{N}$ & $\mathrm{N}$ & $\mathrm{N}$ & $\mathrm{P}$ \\
\hline 21 & MZL & BMB & $\mathrm{N}$ & $\mathrm{N}$ & $\mathrm{N}$ & $\mathrm{N}$ \\
\hline 22 & MZL & BMB & $\mathrm{N}$ & $\mathrm{N}$ & $\mathrm{N}$ & $\mathrm{N}$ \\
\hline 23 & MALT & BMB & $\mathrm{N}$ & $\mathrm{N}$ & $\mathrm{N}$ & $\mathrm{N}$ \\
\hline 24 & HCL & BMB & $\mathrm{N}$ & $\mathrm{N}$ & $\mathrm{N}$ & $\mathrm{N}$ \\
\hline 25 & HCL & BMB & $\mathrm{N}$ & $\mathrm{N}$ & $\mathrm{N}$ & $\mathrm{N}$ \\
\hline 26 & HCL & BMB & $\mathrm{N}$ & $\mathrm{N}$ & $\mathrm{N}$ & $\mathrm{N}$ \\
\hline 27 & Normal & BMB & $\mathrm{N}$ & $\mathrm{N}$ & $\mathrm{N}$ & $\mathrm{N}$ \\
\hline 28 & Normal & BMB & $\mathrm{N}$ & $\mathrm{N}$ & $\mathrm{N}$ & $\mathrm{N}$ \\
\hline 29 & Normal & BMB & $\mathrm{N}$ & $\mathrm{N}$ & $\mathrm{N}$ & $\mathrm{N}$ \\
\hline
\end{tabular}

B-CLL, B-cell chronic lymphocytic leukemia; BMB, bone marrow biopsy; HCL, hairy cell leukemia; LBL, lymphoblastic lymphoma; LN, lymph node; MALT, MALT type lymphoma; MZL, marginal zone lymphoma; N, negative; P, positive.

${ }^{a}$ The number of specimens is the same as the number of patients.

by incubation at $70^{\circ} \mathrm{C}$ for 15 minutes. Finally, any unused hexamers were removed with RNase $\mathrm{H}$ (Ambion, Austin, TX). The primers used to amplify the cyclin D1 transcript are shown in Table 3. The cycling conditions were as follows: $94^{\circ} \mathrm{C}$ for $30 \mathrm{sec}-$ onds; $59^{\circ} \mathrm{C}$ for 30 seconds; and $72^{\circ} \mathrm{C}$ for 1 minute for 30 and 35 cycles. As a control for the RNA and cDNA quality, amplification of a glyceraldehyde phosphate dehydrogenase transcript was used. Samples lacking the amplified glyceraldehyde phosphate dehydrogenase product were not further processed for cyclin D1 PCR.

IHC

The staining conditions for the monoclonal antibodies CD20, CD45RA, CD3, CD45RO, CD23, CD34,
TdT, and CD5 are shown in Table 4. IHC for cyclin D1 was performed on 3- to 4- $\mu$ m-thick paraffin sections with a standard streptavidin-biotin method (22). The sections were placed on positively charged slides. A heat-induced epitope retrieval method was used before immunostaining - that is, sections were placed in $0.01 \mathrm{~m}$ sodium citrate buffer at $\mathrm{pH} 6.0$ and heated in a microwave oven for 10,9 , and 5 minutes. The antibodies to cyclin D1 were purchased from Novocastra Laboratories (Newcastle, England). Reactivity was detected by a StrepABComplex/HRP system employing 3',3'-diaminobenzidine-tetrahydrochloride dihydrate as chromogen (DAKO, Carpinteria, CA). Positive immunostaining was considered to be present when the majority $(>50 \%)$ of the nuclei of neoplastic cells appeared positive, even if the stain was faint. Cytoplas-

TABLE 3. PCR Methods and Primers

\begin{tabular}{|c|c|c|c|}
\hline Target & PCR Template & Primer Sequences ( $5^{\prime}$ to $3^{\prime}$ ) & Reference \\
\hline Cyclin D1 & RNA/cDNA & $\begin{array}{l}\text { F: AACAGATCATCCGCAAACAC } \\
\text { R: TCACACTTGATCACTCTGGA }\end{array}$ & \\
\hline GAPDH & RNA/cDNA & $\begin{array}{l}\text { F: ACTGGCGTCTTCACCACCAT } \\
\text { R: TCACACTTGACACTCTGGA }\end{array}$ & \\
\hline $\mathrm{t}(11 ; 14)$ & DNA (first round) & $\begin{array}{l}\text { F: CTACTGAAGGACTTGTGGGTTGCT } \\
\text { R: TGAGGAGACGGTGACC }\end{array}$ & 13 \\
\hline & PCR product from the first round (nested) & $\begin{array}{l}\text { F: ATAAGGCTGCTGTACACATCGGTG } \\
\text { R: GTGACCAGGGTNCCTTGGCCCCAG }\end{array}$ & \\
\hline c-erb-B2 & DNA & $\begin{array}{l}\text { F: GGGAAAACCGCGGACGCCTG } \\
\text { R: GTCCCTGTGTACGAGCCGCAC }\end{array}$ & \\
\hline
\end{tabular}

GAPDH, glyceraldehyde phosphate dehydrogenase; PCR, polymerase chain reaction. 
TABLE 4. Antibodies and Conditions for Immunohistochemistry on Lymphomas

\begin{tabular}{|c|c|c|c|c|}
\hline Antibody & Pretreatment & Method & Source & Dilution \\
\hline L-26/CD20 & Microwave, CB, pH 6.0 & PAP & DAKO & $1: 30$ \\
\hline Pan-B/CD45R & Microwave, CB, pH 6.0 & PAP & DAKO & $1: 20$ \\
\hline UCHL1/CD45RO & & PAP & DAKO & $1: 50$ \\
\hline CD3 & Microwave, SCB, pH 6.0 & Streptavidin-biotin & Novocastra & $1: 50$ \\
\hline CD5 & Microwave, SCB, pH 6.0 & Streptavidin-biotin & Novocastra & $1: 30$ \\
\hline CD23 & Microwave, SCB, pH 6.0 & Streptavidin-biotin & Novocastra & $1: 30$ \\
\hline CD21 & & PAP & DAKO & $1: 10$ \\
\hline Cyclin D1 & Microwave, SCB, pH 6.0 & Streptavidin-biotin & Novocastra & $1: 20$ \\
\hline CD34 & Microwave, SCB, pH 6.0 & PAP & Novocastra & $1: 30$ \\
\hline TdT & Microwave, EDTA, pH 9.0 & PAP & DAKO & $1: 10$ \\
\hline
\end{tabular}

CB, citrate buffer; EDTA, ethylene diamine tetra-acetic acid; SCB, sodium citrate buffer.

mic staining was not considered to represent evidence of immunopositivity.

\section{DNA Extraction and Nested PCR for $\mathrm{t}(11 ; 14)$}

DNA extraction was performed from six to eight $10-\mu \mathrm{m}$-thick paraffin sections with the QIAamp DNA mini kit (Qiagen, Valencia, CA), according to the manufacturer's instructions. A nested PCR pro- tocol was employed that used the primers shown in Table 3, as reported by Lasota et al. (13). The cycling conditions were as follows: $94^{\circ} \mathrm{C}$ for 3 minutes, $\left(94^{\circ} \mathrm{C}\right.$ for $30 \mathrm{~s}, 59^{\circ} \mathrm{C}$ for $1 \mathrm{~min}, 72^{\circ} \mathrm{C}$ for $\left.1 \mathrm{~min}\right) \times 30$, and $72^{\circ} \mathrm{C}$ for 7 minutes. Amplification of a c-erbB-2 gene sequence was used as DNA quality control. Samples negative for the c-erbB-2 product were not further processed for the nested PCR protocol.

TABLE 5. RT-PCR, PCR, ISH, and IHC Results in the Study Group

\begin{tabular}{|c|c|c|c|c|c|c|}
\hline $\begin{array}{c}\text { Patient } \\
\text { No. }\end{array}$ & Tissue Specimen & MCL & RT-PCR & $\mathrm{t}(11 ; 14)$ & ISH & $\mathrm{IHC}^{a}$ \\
\hline 1 & LN & $\mathrm{CV}$ & $\mathrm{P}$ & ND & $P$ & $\mathrm{~N}$ \\
\hline 2 & LN & CV & ND & $\mathrm{P}$ & $\mathrm{P}$ & $\mathrm{P}$ \\
\hline 3 & $\mathrm{LN}$ & $\mathrm{CV}$ & ND & $\mathrm{P}$ & ND & $\mathrm{P}$ \\
\hline 4 & $\mathrm{LN}$ & $\mathrm{CV}$ & ND & $\mathrm{P}$ & $\mathrm{P}$ & $\mathrm{N}$ \\
\hline 5 & $\mathrm{LN}$ & $\mathrm{CV}$ & $\mathrm{P}$ & ND & $\mathrm{P}$ & $\mathrm{P}$ \\
\hline 6 & $\mathrm{LN}$ & $\mathrm{CV}$ & $\mathrm{P}$ & ND & $\mathrm{P}$ & $\mathrm{N}$ \\
\hline 7 & $\mathrm{LN}$ & $\mathrm{CV}$ & P & $\mathrm{N}$ & $\mathrm{P}$ & $\mathrm{P}$ \\
\hline 8 & $\mathrm{LN}$ & $\mathrm{CV}$ & $\mathrm{P}$ & $\mathrm{P}$ & $\mathrm{P}$ & $\mathrm{P}$ \\
\hline 9 & $\mathrm{LN}$ & $\mathrm{CV}$ & ND & $\mathrm{N}$ & $P$ & $\mathrm{P}$ \\
\hline 10 & $\mathrm{LN}$ & $\mathrm{CV}$ & $\mathrm{P}$ & $\mathrm{P}$ & $\mathrm{P}$ & $\mathrm{P}$ \\
\hline 11 & LN & $\mathrm{CV}$ & $\mathrm{N}$ & ND & $\mathrm{N}$ & $\mathrm{N}$ \\
\hline 12 & $\mathrm{LN}$ & $\mathrm{CV}$ & $\mathrm{N}$ & $\mathrm{N}$ & $\mathrm{N}$ & $\mathrm{N}$ \\
\hline 13 & $\mathrm{LN}$ & $\mathrm{CV}$ & $\mathrm{P}$ & $\mathrm{N}$ & $\mathrm{N}$ & $\mathrm{N}$ \\
\hline 14 & LN & CV & ND & $\mathrm{N}$ & $\mathrm{P}$ & $\mathrm{P}$ \\
\hline 15 & Palate $^{b}$ & $\mathrm{CV}$ & $\mathrm{P}$ & $\mathrm{N}$ & $\mathrm{P}$ & $\mathrm{P}$ \\
\hline 16 & $\mathrm{LN}$ & BV & ND & ND & $\mathrm{N}$ & $\mathrm{N}$ \\
\hline 17 & $\mathrm{LN}$ & BV & $\mathrm{P}$ & $\mathrm{P}$ & $\mathrm{P}$ & $\mathrm{P}$ \\
\hline 18 & $\mathrm{LN}$ & $\mathrm{BV}$ & $\mathrm{P}$ & $\mathrm{P}$ & $\mathrm{P}$ & $\mathrm{P}$ \\
\hline 19 & $\mathrm{LN}$ & $\mathrm{BV}$ & $\mathrm{P}$ & $\mathrm{N}$ & $\mathrm{P}$ & $\mathrm{P}$ \\
\hline 20 & LN & BV & P & $\mathrm{N}$ & $\mathrm{P}$ & $\mathrm{P}$ \\
\hline 21 & LN & BV & $\mathrm{P}$ & $\mathrm{P}$ & $\mathrm{P}$ & $\mathrm{P}$ \\
\hline 22 & $\mathrm{LN}$ & BV & $\mathrm{P}$ & $\mathrm{P}$ & $\mathrm{P}$ & $\mathrm{P}$ \\
\hline 23 & $\mathrm{LN}$ & BV & $\mathrm{P}$ & ND & ND & ND \\
\hline 24 & Nasopharynd $^{b}$ & $\mathrm{BV}$ & ND & $\mathrm{N}$ & $\mathrm{P}$ & $\mathrm{P}$ \\
\hline 25 & Skin & $\mathrm{BV}$ & ND & $\mathrm{N}$ & $\mathrm{P}$ & $\mathrm{N}$ \\
\hline 26 & BMB & $\mathrm{CV}$ & ND & $\mathrm{N}$ & ND & $\mathrm{P}$ \\
\hline 26 & Small intestine & $\mathrm{CV}$ & $\mathrm{P}(\mathrm{ni})$ & $\mathrm{N}$ & ND & ND \\
\hline 26 & Stomach & $\mathrm{CV}$ & $\mathrm{P}(\mathrm{ni})$ & $\mathrm{P}$ & $\mathrm{P}$ & $\mathrm{P}$ \\
\hline 27 & $\mathrm{LN}$ & $\mathrm{CV}$ & $\mathrm{P}$ & $\mathrm{N}$ & $\mathrm{P}$ & $\mathrm{N}$ \\
\hline 27 & Liver & $\mathrm{CV}$ & $\mathrm{P}(\mathrm{ni})$ & $\mathrm{P}$ & $\mathrm{P}$ & $\mathrm{N}$ \\
\hline 27 & Spleen & $\mathrm{CV}$ & $\mathrm{P}$ & $\mathrm{P}$ & $\mathrm{P}$ & $\mathrm{N}$ \\
\hline 28 & Stomach & $\mathrm{CV}$ & ND & $\mathrm{P}$ & $\mathrm{P}$ & $\mathrm{P}$ \\
\hline 28 & BMB & CV & ND & $\mathrm{P}$ & $\mathrm{P}$ & $\mathrm{N}$ \\
\hline 29 & $\mathrm{LN}$ & $\mathrm{CV}$ & ND & $\mathrm{P}$ & $\mathrm{P}$ & $\mathrm{P}$ \\
\hline 29 & BMB & $\mathrm{CV}$ & ND & $\mathrm{P}$ & $\mathrm{P}$ & $\mathrm{P}$ \\
\hline 30 & Stomach & $\mathrm{CV}$ & $\mathrm{P}(\mathrm{ni})$ & $\mathrm{P}$ & $\mathrm{P}$ & $\mathrm{P}$ \\
\hline 30 & $\mathrm{LN}$ & $\mathrm{CV}$ & ND & $\mathrm{P}$ & $\mathrm{P}$ & $\mathrm{P}$ \\
\hline
\end{tabular}

BV, blastoid variant; CV, centrocytoid variant; IHC, immunohistochemistry; ISH, in situ hybridization; LN, lymph node; MCL, mantle cell lymphoma; $\mathrm{N}$, negative; ND, not done; $\mathrm{P}$, positive; $\mathrm{PCR}$, polymerase chain reaction; $\mathrm{P}(\mathrm{ni})$, patients positive for cyclin D1 but noninformative because of tissue elements normally expressing this gene; RT-PCR, reverse transcriptase-PCR.

${ }^{a}$ Nuclear staining in $>50 \%$ of the neoplastic cell population.

${ }^{b}$ Without epithelium. 
Specificity of the nested PCR products was confirmed by DNA sequencing.

\section{RESULTS}

The data obtained by the methods evaluated are presented in Tables 5 and 6. ISH was performed in 33 specimens from 28 patients in the study group; $24(86 \%)$ were positive for cyclin D1 mRNA. Positivity was evident as strong blue perinuclear staining of the neoplastic lymphoid cells (Fig. 2A). In the extranodal cases involving epithelial tissues, such as stomach, intestine, and skin, basally located epithelial cells were also strongly positive for cyclin D1 mRNA (Fig. 2B). Occasional hepatocytes were also found to be positive. None of the patients in the control group were found to be positive for cyclin D1 mRNA. However, in extranodal lymphomas, epithelial cells, such as those in Patients 12 and 13 of the control group (Table 6), strongly expressed cyclin D1 mRNA.

RT-PCR for the cyclin D1 transcript was performed in 23 specimens from 20 patients of the study group (excluded from the total number of 37 specimens were three bone marrow biopsies, eight lymph node specimens, and two extranodal speci- mens with inadequate material, as well as two specimens with degraded RNA [Table 5]). RT-PCR was also performed in 16 patients of the control group (excluded from the total of 29 specimens were 12 paraffin-embedded bone marrow biopsies and one MZL case with inadequate material [Table 6]).

The cyclin D1 product was present in 21 of 23 samples (92\% positivity) in the study group (Fig. $3 \mathrm{~A})$. However, 4 of these 23 specimens represented MCL in extranodal tissues where cyclin D1 is normally expressed (gastrointestinal mucosa and liver). Hence these four specimens were also considered to be noninformative, although positive. Thus RTPCR was considered as informative for the cyclin D1 transcript in 19 specimens. Seventeen of these were positive for the cyclin D1 transcript, corresponding to 16 of 18 patients (89\% positivity). PCR amplification for 35 cycles increased product yield but did not further increase the number of positive samples.

In the control group, two patients with MALT lymphoma were found to be positive for the cyclin D1 product after 30 cycles of PCR amplification; however, these samples contained gastric mucosa that normally expresses cyclin D1 and were hence considered to be noninformative. All informative

TABLE 6. RT-PCR, PCR, ISH, and IHC Results in the Control Group

\begin{tabular}{|c|c|c|c|c|c|c|c|}
\hline \multirow{2}{*}{$\begin{array}{c}\text { Patient } \\
\text { No. }\end{array}$} & \multirow{2}{*}{ Diagnosis } & \multirow{2}{*}{ Tissue } & \multicolumn{2}{|c|}{ RT-PCR } & \multirow{2}{*}{$\mathrm{t}(11 ; 14)$} & \multirow{2}{*}{ ISH } & \multirow{2}{*}{$\mathrm{IHC}^{a}$} \\
\hline & & & 30 Cycles & 35 Cycles & & & \\
\hline 1 & B-CLL & $\mathrm{LN}$ & $\mathrm{N}$ & $\mathrm{N}$ & $\mathrm{N}$ & ND & $\mathrm{N}$ \\
\hline 2 & B-CLL & $\mathrm{LN}$ & $\mathrm{N}$ & $\mathrm{P}$ & $\mathrm{N}$ & ND & $\mathrm{N}$ \\
\hline 3 & B-CLL & $\mathrm{LN}$ & $\mathrm{N}$ & $\mathrm{N}$ & $\mathrm{N}$ & ND & $\mathrm{N}$ \\
\hline 4 & B-CLL & $\mathrm{LN}$ & $\mathrm{N}$ & $\mathrm{P}$ & $\mathrm{P}$ & ND & $\mathrm{N}$ \\
\hline 5 & B-CLL & $\mathrm{LN}$ & $\mathrm{N}$ & $\mathrm{N}$ & $\mathrm{N}$ & ND & $\mathrm{N}$ \\
\hline 6 & B-CLL & $\mathrm{LN}$ & $\mathrm{N}$ & $\mathrm{N}$ & $\mathrm{N}$ & ND & $\mathrm{N}$ \\
\hline 7 & B-CLL & $\mathrm{LN}$ & $\mathrm{N}$ & $\mathrm{N}$ & $\mathrm{N}$ & $\mathrm{N}$ & $\mathrm{N}$ \\
\hline 8 & MZL & $\mathrm{LN}$ & $\mathrm{N}$ & $\mathrm{N}$ & $\mathrm{N}$ & $\mathrm{N}$ & $\mathrm{N}$ \\
\hline 9 & MZL & Spleen & $\mathrm{N}$ & $\mathrm{P}$ & $\mathrm{N}$ & $\mathrm{N}$ & $\mathrm{N}$ \\
\hline 10 & MZL & Spleen & ND & ND & $\mathrm{N}$ & $\mathrm{N}$ & $\mathrm{N}$ \\
\hline 11 & MALT & $\mathrm{LN}$ & $\mathrm{N}$ & $\mathrm{N}$ & $\mathrm{N}$ & $\mathrm{N}$ & $\mathrm{N}$ \\
\hline 12 & MALT & Gastric & $\mathrm{P}(\mathrm{ni})$ & $\mathrm{P}(\mathrm{ni})$ & $\mathrm{N}$ & $\mathrm{N}$ & $\mathrm{N}$ \\
\hline 13 & MALT & Gastric & $\mathrm{P}(\mathrm{ni})$ & $\mathrm{P}(\mathrm{ni})$ & $\mathrm{N}$ & $\mathrm{N}$ & $\mathrm{N}$ \\
\hline 14 & LBL & LN & $\mathrm{N}$ & $\mathrm{P}$ & $\mathrm{N}$ & $\mathrm{N}$ & $\mathrm{N}$ \\
\hline 15 & LBL & $\mathrm{LN}$ & $\mathrm{N}$ & $\mathrm{N}$ & $\mathrm{N}$ & ND & $\mathrm{N}$ \\
\hline 16 & LBL & $\mathrm{LN}$ & $\mathrm{N}$ & $\mathrm{N}$ & $\mathrm{N}$ & ND & $\mathrm{N}$ \\
\hline 17 & LBL & $\mathrm{LN}$ & $\mathrm{N}$ & $\mathrm{N}$ & $\mathrm{N}$ & ND & $\mathrm{N}$ \\
\hline 18 & B-CLL & $\mathrm{BMB}$ & ND & ND & ND & $\mathrm{N}$ & $\mathrm{N}$ \\
\hline 19 & B-CLL & $\mathrm{BMB}$ & ND & ND & ND & $\mathrm{N}$ & $\mathrm{N}$ \\
\hline 20 & B-CLL & BMB & ND & ND & ND & $\mathrm{N}$ & $\mathrm{N}$ \\
\hline 21 & MZL & $\mathrm{BMB}$ & ND & ND & ND & $\mathrm{N}$ & $\mathrm{N}$ \\
\hline 22 & MZL & BMB & ND & ND & ND & $\mathrm{N}$ & $\mathrm{N}$ \\
\hline 23 & MALT & $\mathrm{BMB}$ & ND & ND & ND & $\mathrm{N}$ & $\mathrm{N}$ \\
\hline 24 & HCL & $\mathrm{BMB}$ & ND & ND & ND & $\mathrm{N}$ & $\mathrm{N}$ \\
\hline 25 & HCL & $\mathrm{BMB}$ & ND & ND & ND & $\mathrm{N}$ & $\mathrm{N}$ \\
\hline 26 & HCL & $\mathrm{BMB}$ & ND & ND & ND & $\mathrm{N}$ & $\mathrm{N}$ \\
\hline 27 & Normal & $\mathrm{BMB}$ & ND & ND & ND & $\mathrm{N}$ & $\mathrm{N}$ \\
\hline 28 & Normal & BMB & $\mathrm{ND}$ & ND & ND & $\mathrm{N}$ & $\mathrm{N}$ \\
\hline 29 & Normal & $\mathrm{BMB}$ & ND & ND & ND & $\mathrm{N}$ & $\mathrm{N}$ \\
\hline
\end{tabular}

B-CLL, B-cell chronic lymphocytic leukemia; BMB, bone marrow biopsy; HCL, hairy cell leukemia; IHC, immunohistochemistry; ISH, in situ hybridization; LBL, lymphoblastic lymphoma; LN, lymph node; MALT, MALT type lymphoma; MZL, marginal zone lymphoma; N, negative; ND, not done; $\mathrm{P}$, positive; $\mathrm{PCR}$, polymerase chain reaction; $\mathrm{P}(\mathrm{ni})$, patients positive for cyclin $\mathrm{D} 1$ but noninformative because of tissue elements normally expressing this gene; RT-PCR, reverse transcriptase-PCR.

${ }^{a}$ Nuclear staining in $>50 \%$ of the neoplastic cell population. 

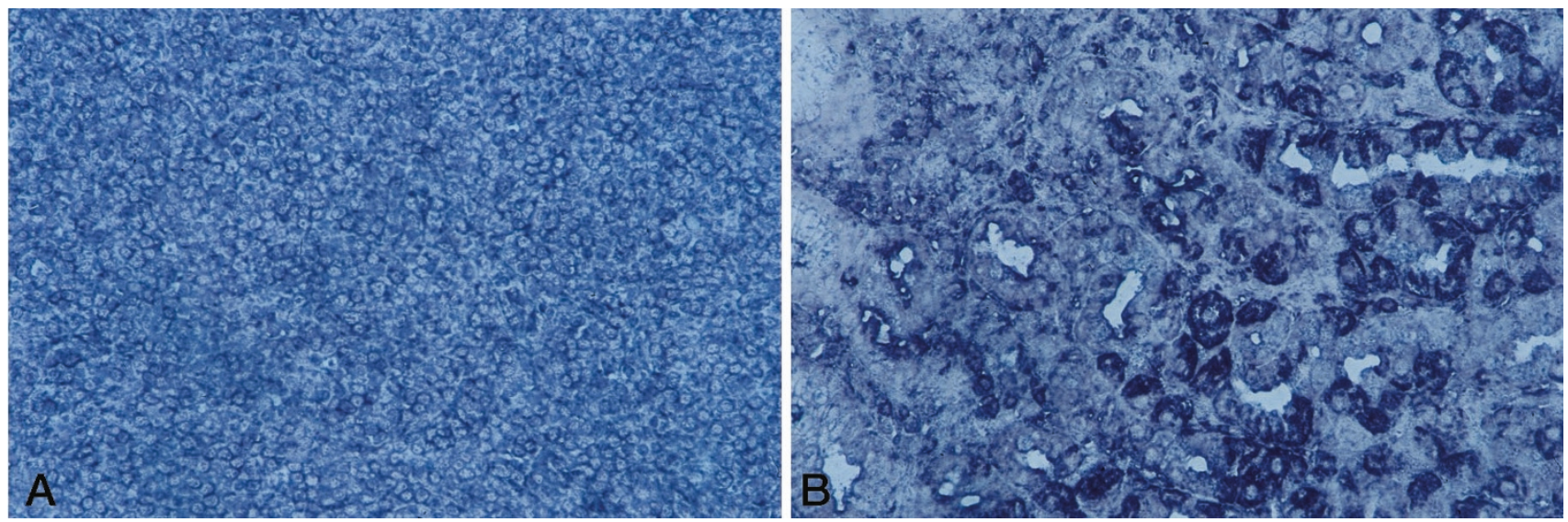

FIGURE 2. In situ hybridization for cyclin D1 mRNA. A, mantle cell lymphoma cells infiltrating a lymph node show diffuse perinuclear positivity. Original magnification, 400×. B, in this gastric biopsy, epithelial cells also express cyclin D1 mRNA. Original magnification, 400×.

patients $(n=15)$ were negative for the cyclin D1 product under the same amplification conditions (Table 6, Fig. 3C). However, after 35 amplification cycles, a faint band was present in four informative patients, including two cases of B-CLL, one case of MZL, and one case of lymphoblastic lymphoma (Table 6, Fig. 3D). The specificity of the RT-PCR products was verified by sequencing of five randomly selected patients (three from the study group and two from the control group).

IHC for cyclin D1 was performed in 35 specimens from 29 patients. Twenty (69\%) patients exhibited

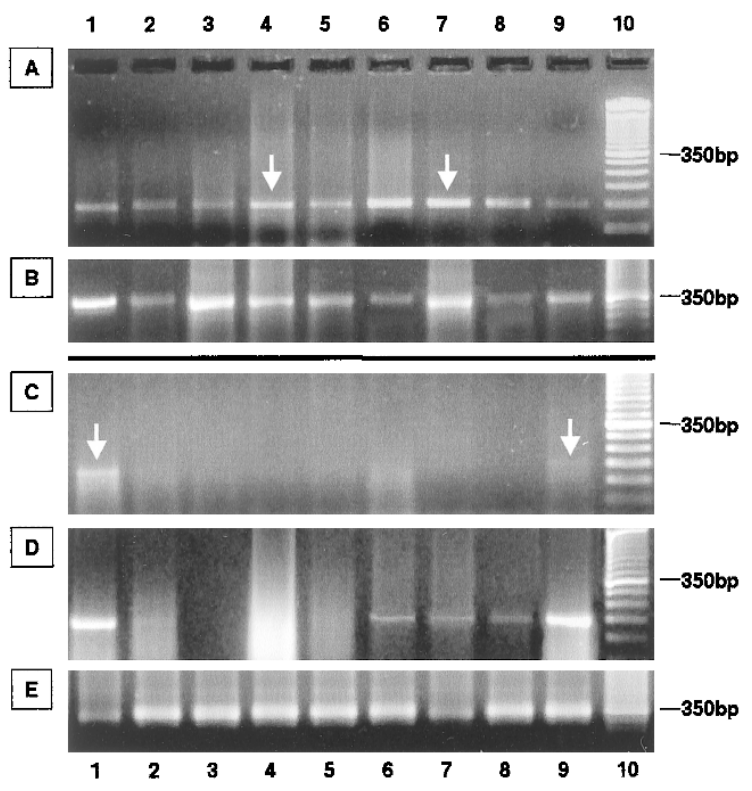

FIGURE 3. Reverse transcriptase-polymerase chain reaction results for cyclin D1 (A, C, D) and for glyceraldehyde phosphate dehydrogenase (RNA control; B, E). Representative patients from the study group positive for the cyclin D1 transcript (30 amplification cycles) are shown in A, lanes 1-9. Representative patients from the control group checked for cyclin D1 expression, amplified for 30 and 35 cycles, are shown in $\mathbf{C}$ and $\mathbf{D}$, respectively, lanes 1-9. Lane 10: 50-base pair (bp; in A, B, C, D) and 10-bp ladder (in E). Positive but noninformative cases containing tissue elements normally expressing the cyclin D1 gene are indicated (arrows). positive nuclear staining for Cyclin D1 protein in more than $50 \%$ of the neoplastic population (Fig. 1C). Nuclear staining for this antigen was not observed in any case of the control group. However, four patients in the control group (cases of MZL and B-CLL) exhibited cytoplasmic staining.

Nested PCR for the identification of $t(11 ; 14)$ was performed in 31 specimens (24 patients); the translocation was found in 14 (58\%) patients, including two bone marrow biopsies (Fig. 4). By contrast, only one patient in the control group (B-CLL) was found to be positive for $t(11 ; 14)$. The specificity of the nested PCR products was confirmed by sequencing, which was performed in eight randomly selected patients.

\section{DISCUSSION}

MCL is a lymphoproliferative disorder derived from a subset of naive pregerminal center cells, which is characterized by a nodular or diffuse proliferation of atypical lymphoid cells with a monoclonal B-cell phenotype. Two types have been described: the centrocytoid and the blastoid. Cases of

$\begin{array}{lllllllllllllllll}1 & 2 & 3 & 4 & 5 & 6 & 7 & 8 & 9 & 10 & 11 & 12 & 13 & 14 & 15 & 16 & 17\end{array}$ A
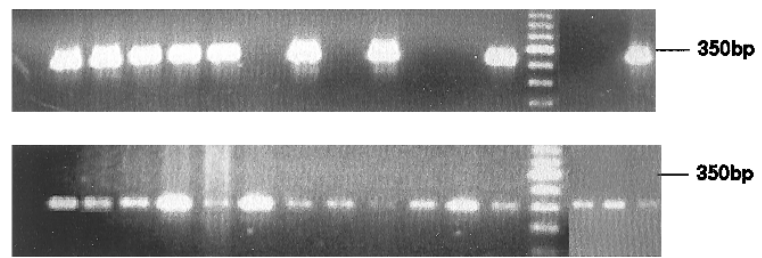

FIGURE 4. A, Polymerase chain reaction (PCR) results for the $t(11$; 14) translocation. B, the nested PCR protocol was performed after the amplification for a c-erb-B2 sequence for the DNA control.

Representative cases both from the study and control groups are shown. Lane 1: negative control; lanes 2-13: mantle cell lymphoma cases; lane 14: 50-base pair (bp) ladder; lane 15: marginal zone lymphoma; lane 16: lymphoblastic lymphoma; lane 17: B-cell chronic lymphocytic leukemia, representing the only positive case in the control group. 
the blastoid type have a higher proliferative activity and a more aggressive clinical evolution (23). MCL usually affects elderly men, who present with advanced disease and frequent extranodal involvement. The clinical evolution is relatively aggressive, with poor response to conventional therapeutic regimens and a median survival of 3 to 4 years. The identification of a typical MCL immunohistochemical profile, which includes CD20, CD45RA, and cyclin D1 positivity, has made diagnosis more accurate; however, IHC performed on paraffin sections is not always helpful in differentiating between MCL and low-grade B-cell lymphomas, mostly because of variable staining depending on fixation (16). Therefore, molecular methods may be needed for definitive diagnosis.

The hallmark of MCL is $t(11 ; 14)$ (q13;q32), leading to overexpression of cyclin D1, which plays an important pathogenetic role in lymphomagenesis (9). Cyclin D1 belongs to the family of D-type cyclin proteins, which function primarily by regulating the activity of certain protein kinases in the G1 phase of the cell cycle. Maximum expression of cyclin D1 occurs at a critical point in mid- to late G1 phase. Immunohistochemical positivity for cyclin D1 characterizes MCL but has also been found in small numbers of cases of other lymphoproliferative disorders, such as aggressive B-CLL, prolymphocytic leukemia, hairy cell leukemia, and plasma cell myeloma (14, 24-26). Cyclin D1 immunopositivity was demonstrated in $69 \%$ of our study group patients, whereas all specimens in the control group were found to be negative for this marker. This rate confirms previous studies that used monoclonal or polyclonal antibodies on paraffin-embedded lymph nodes and bone marrow biopsies, as well as frozen tissues with MCL (10, 14, 27-29).

The aim of our study was to evaluate the usefulness of ISH and RT-PCR for cyclin D1 mRNA in the diagnosis of MCL. In line with the findings of other researchers (16), we showed that RT-PCR applied on paraffin-embedded tissues is a sensitive method for the demonstration of cyclin D1 mRNA, because $89 \%$ of our study group patients were found to be positive. A problem with the interpretation of the RT-PCR results is specificity. In this setting, specificity has two aspects: first, cyclin D1 expression by non-MCL neoplastic cells (16-18); and second, cyclin D1 expression by normal cells included in any given tissue sample $(30,31)$.

Semiquantitative PCR methods have been used to overcome the specificity burden of the first aspect (16-18). In our study, a semiquantitative approach was not deemed necessary because no cases of the non-MCL control group were considered positive under the stringent PCR conditions used. In fact, when we initially applied lower annealing temperatures $\left(55^{\circ} \mathrm{C}\right)$ and higher cycle numbers (up to 40 ), more than $50 \%$ of the control group patients were positive for the cyclin D1 transcript (data not shown). However, this phenomenon is probably related to the supersensitivity of PCR after prolonged cycling under favorable conditions. Therefore, we think that the stringent PCR protocol used in this study provides an adequate means as far as diagnosis of MCL in lymph node material is concerned. Obviously, a further improvement would be to use real quantitative PCR protocols, such as those with real-time amplification; nevertheless, there are no such reports in the literature at present, whereas cut-off values for cyclin D1 mRNA levels diagnostic for MCL have still to be established.

The second aspect of specificity regarding cyclin D1 RT-PCR, as mentioned above, refers to falsepositive findings when extranodal tissues containing various types of epithelia are evaluated. It must be taken into consideration that PRAD-1/cyclin D1 is a normally expressed gene during the G1/S transition in the majority of cell types, including proliferating epithelia (30) and fibroblasts (31). In contrast, cyclin D1 is not expressed in normal lymphoid tissues $(16,17,30)$ and it does not seem to be necessary for cell cycle progression in normal lymphocytes, which instead use other cyclins (17, $31,32)$. Thus we suggest that RT-PCR for cyclin D1 expression should not be used in the diagnosis of extranodal MCL, unless the tissue specimen, from which RNA is extracted, is homogeneously composed of neoplastic cells. The presence of native tissue elements normally expressing cyclin D1 during proliferation, such as epithelia of the gastrointestinal tract, nasopharynx, or skin, should exclude the processing of these specimens for cyclin D1 expression evaluation by RT-PCR, either with or without quantitation. This problem may be overcome by careful laser-assisted microdissection of the target neoplastic cells; however, protocols involving microdissection of neoplastic lymphoid populations from paraffin-embedded tissues and subsequent quantitative RT-PCR are still under evaluation.

These problems of specificity arising during the interpretation of the RT-PCR results are adequately answered by ISH, which is of great value in assessing cell type specific cyclin D1 expression on paraffin-embedded tissues. The only previous study on cyclin D1 ISH performed on paraffin-embedded tissues is that of Williams et al. (20), where all five cases of MCL were positive. In our study, ISH was performed in 33 samples from 28 patients; 24 (86\%) were positive. ISH was applied successfully on all types of paraffin-embedded specimens, including bone marrow biopsies. Its sensitivity in the detection of MCL was superior to IHC (86\% versus 69\%). In comparison to the stringent RT-PCR protocol 
used, ISH missed only one case (Patient 13; Table $5)$, where the cyclin D1 transcript was detected by RT-PCR. The major advantage of ISH in comparison to the PCR method is in regard to specificitythat is, the ability to morphologically identify the cells that express cyclin D1. Thus cyclin D1 mRNA could be detected in MCL cells, as well as in normal cells in extranodal cases, but not in non-MCL cells. The lack of positivity in our control group probably reflects the relatively low amounts of this transcript reported in lymphomas other than MCL (16). Clearly more studies are needed to confirm our data; nevertheless, at present, ISH seems to be a useful technique for the detection of cyclin D1 expression in MCL diagnosis. In addition, this method can be used to detect minimal residual disease in bone marrow biopsies.

In this study, we also used a nested PCR protocol to detect the presence of $t(11 ; 14)$ (q13;q32). Seventy percent to $80 \%$ of the breakpoints involved in this translocation are located in the major translocation cluster (29). Breakpoints outside the major translocation cluster cannot be detected by PCR; this is why the reported incidence of $t(11 ; 14)$, as demonstrated by PCR, is relatively low (i.e., in the range of $40 \%$ ) (13). In our material, the detection rate was $58 \%$. However, this marker was fairly specific because only one patient (B-CLL) of the control group was also found to be positive. All patients positive for the $t(11 ; 14)$ were found to express cyclin D1, in accordance with the proposed implication for this translocation at the functional level.

We conclude that ISH and stringent RT-PCR for cyclin D1 mRNA are sensitive methods for the confirmation of MCL diagnosis. However, the application of RT-PCR bears certain restrictions, as mentioned above, whereas quantitative real-time protocols on microdissected tissues are on the way to being established. Therefore, we suggest that ISH, which provides the ability to correlate the molecular findings with morphology, should be the method of choice for the practicing pathologist to assess cyclin D1 expression in MCL diagnosis.

\section{REFERENCES}

1. Weisenburger DD, Kim H, Rappaport H. Mantle-zone lymphoma: a follicular variant of intermediate lymphocytic lymphoma. Cancer 1982;49:1429-38.

2. Weisenburger DD, Armitage JO. Mantle cell lymphoma-an entity comes of age. Blood 1996;87:4483-94.

3. Wong KF, Chan JK, So JC, Yu PH. Mantle cell lymphoma in leukemic phase: characterization of its broad cytologic spectrum with emphasis on the importance of distinction from other chronic lymphoproliferative disorders. Cancer 1999; 86:850-7.

4. Campo E, Jaffe ES. Mantle cell lymphoma: accurate diagnosis yields new clinical insights. Arch Pathol Lab Med 1996; 120:12-4.
5. Argatoff LH, Connors JM, Klassa RJ, Horsmann DE, Gascoyne RD. Mantle cell lymphoma: a clinicopathologic study of 80 cases. Blood 1997;89:2067-87.

6. Harris NL, Jaffe ES, Stein H, Banks PM, Chan JKC, Cleary ML, et al. A revised European-American classification of lymphoid neoplasms: a proposal from the International Lymphoma Study Group. Blood 1994;84:1361-92.

7. Jaffe ES, Harris NL, Diebold J, Müller-Hermeling HK. World Health Organization classification of neoplastic disease of the hematopoetic and lymphoid tissues: a progress report. Am J Clin Pathol 1998;110(Suppl 1):S8-S12.

8. Ott MM, Helbing A, Ott G, Bartek J, Fisher L, Durr A, et al. bcl-1 rearrangement and cyclin D1 protein expression in mantle cell lymphoma. J Pathol 1996;179:238-42.

9. Oka K, Ohno T, Yamaguchi M, Mahmud N, Miwa H, Kita K, et al. PRAD-1/cyclin D1 gene overexpression in mantle cell lymphoma. Leuk Lymphoma 1996;21:37-42.

10. Hashimoto Y, Nakamura N, Kuze T, Abe M, Wakasa H. Intranuclear expression of cyclin D1 protein as a useful prognostic marker for mantle cell lymphoma. Fukushima J Med Sci 1997;43:87-98.

11. Cuneo A, Bigoni R, Rigolin GM, Roberti MG, Bardi A, Piva N, et al. Cytogenetic profile of lymphoma of follicle mantle lineage: correlation with clinicobiologic features. Blood 1999;93:1372-80.

12. Vaandrager JW, Schuuring E, Zwikstra E, de Boer CJ, Kleiverda KK, Van Krieken JH, et al. Direct visualization of dispersed 11q13 chromosomal translocations in mantle cell lymphoma by multicolor DNA fiber fluorescence in situ hybridization. Blood 1996;88:1177-82.

13. Lasota J, Franssila K, Koo CH, Miettinen M. Molecular diagnosis of mantle cell lymphoma in paraffin embedded tissue. Mod Pathol 1995;9:361-6.

14. Vasef MA, Medeiros LJ, Yospur LS, Sun NCJ, McCourty A, Brynes RK. Cyclin D1 protein in multiple myeloma and plasmacytoma: an immunohistochemical study using fixed paraffin embedded tissue sections. Mod Pathol 1997;10:92732.

15. Jadajel D, Matutes E, Dyer MJS, Brito-Babapulle V, Khohkar MT, Oscier D, et al. Splenic lymphoma with villous lymphocytes: analysis of bcl-1 rearrangements and expression of cyclin d1 gene. Blood 1994;83:3664-71.

16. Ives Aguilera NS, Bijwaard KE, Dunkan B, Krafft AE, Chu WS, Abbondanzo SL, et al. Differential expression of cyclin D1 in mantle cell lymphoma and other non-Hodgkin lymphomas. Am J Pathol 1998;153:1969-76.

17. Uchimaru K, Taniguchi T, Yoshikawa M, Asano S, Arnold A, Fujita T, et al. Detection of cyclin D1 (bcl-1, PRAD1) overexpression by a simple competitive reverse transcription-polymerase chain reaction assay in $t(11 ; 14)(q 13 ; q 32)$-bearing B-cell malignancies and/or mantle cell lymphoma. Blood 1997;89:965-74.

18. Sola B, Salaun V, Ballet JJ, Troussard X. Transcriptional and post-transcriptional mechanisms induce cyclin D1 overexpression in B-chronic lymphoproliferative disorders. Int J Cancer 1999;83:230-4.

19. de Boer CJ, Van Krieken JHJM, Kluin-Nelemans HC, Kluin PM, Schuurin E. Cyclin D1 messenger RNA overexpression as a marker for mantle cell lymphoma. Oncogene 1995;10: 1833-40.

20. Williams ME, Nichols GE, Swerdlow SH, Stoler MH. In situ hybridization detection of cyclin D1 mRNA in centrocytic/ mantle cell lymphoma. Annu Oncol 1995;6:297-9.

21. Zukerberg LR, Yang WI, Arnold A, Harris NL. Cyclin D1 expression in non-Hodgkin's lymphomas. Detection by immunohistochemistry. Am J Clin Pathol 1995;103:756-60.

22. Wood GS, Warnke R. Suppression of endogenous avidinbinding activity in tissues and its relevance to biotin-avidin detection systems. J Histochem Cytochem 1981;29:1196-204. 
23. Campo E, Raffeld M, Jaffe ES. Mantle cell lymphoma. Semin Hematol 1999;36:115-27.

24. Kobayashi H, Saito H, Kitano K, Kiyosawa K, Gaun S, Aoki K, et al. Overexpression of the PRAD1 oncogene in a patient with multiple myeloma and $t(11 ; 14)(q 13 ; q 32)$. Acta Haematol 1995;94:199-203.

25. Swerdlow SH, Yang WI, Zukerberg LR, Harris NL, Arnold A, Williams ME. Expression of cyclin D1 protein in centrocytic/ mantle cell lymphomas with and without rearrangements of bcl-1/cyclin D1 gene. Hum Pathol 1995;26:999-1004.

26. Yang WI, Zukerberg LR, Motokura T, Arnold A, Harris NL. Cyclin D1 (Bcl-1, PRAD1) protein expression in low-grade B-cell lymphomas and reactive hyperplasia. Am J Pathol 1994;145:86-96.

27. Banno S, Yoshikawa K, Nakamura S, Yamamoto K, Seito T, Takahashi T, et al. Monoclonal antibody against PRAD1/cyclin D1 stains nuclei of tumors cells with translocation or amplification at BCL-1 locus. Jpn J Cancer Res 1994;85:918-26.
28. Singh N, Wright DH. The value of immunohistochemistry on paraffin wax embedded tissue sections in the differentiation of small lymphocytic and mantle cell lymphomas. J Clin Pathol 1997;50:16-21.

29. Rimokh R, Berger F, Bastard C, Klein B, French M, Archimbaud E, et al. Rearrangement of CCND1 (BCL1/PRAD1) 3' untranslated region in mantle cell lymphomas and $\mathrm{t}(11 \mathrm{q} 13)$ associated leukemias. Blood 1994;83:3689-96.

30. Bartkova J, Lukas J, Strauss M, Bartek J. Cell cycle-related variation and tissue-restricted expression of human cyclin D1 protein. J Pathol 1994;172:237-45.

31. Juan G, Gong J, Traganos F, Darzynkiewicz Z. Unscheduled expression of cyclins D1 and D3 in human tumour cell lines. Cell Prolif 1996;29:259-66.

32. Furukawa Y, Kikuchi J, Nakamura M, Iwase S, Yamada H, Matsuda M. Lineage-specific regulation of cell cycle control gene expression during haematopoietic cell differentiation. Br J Haematol 2000;110:663-73.

\section{Book Review}

\section{Scheuer PJ, Lefkowitch JH: Liver Biopsy Inter- pretation, Sixth Edition, 381 pp, London, W.B. Saunders, 2000 (\$95.00).}

In the foreword of this beautiful, small book, Professor Dame Sheila Sherlock calls it the liver biopsy bible. Personally, if you allow me to counter with another ecclesiastical metaphor, I would prefer to call it a breviary-in my dictionary defined as the book of daily prayers. Something to be read for inspiration and guidance, or, if lost, so that you could regain the road to the greater truth (read 'correct diagnosis'), I imagine.

If you are a resident, or a practicing diagnostic pathologist in need of a readable, modern, no-nonsense hepatopathology text, this book is almost ideally suited for you. Compact yet comprehensive, it covers essentially everything you need to know from $\mathrm{A}$ to $\mathrm{Z}$ about interpreting liver biopsies. The sixth edition is a fully revised and somewhat expanded version of its predecessor. Beautifully written, lapidary but to the point, it deals with all major liver diseases, i.e., those accounting for at least $90 \%$ of the routine material. For the sake of completeness, the authors mention many less common entities, but for more details you will have to consult references listed at the end of each chapter. These references are up-to-date and well-chosen.
The text is illustrated with instructive photographs, most of which are in full color. The photographs are of enviably high quality and are reproduced in nicely balanced colors. A new chapter on aspiration cytology has been added, in keeping with the new diagnostic trends. This old fashioned adherent of classic histopathology wondered whether this was really needed. In the chapter on liver transplantation, nevertheless, I found enough assurances that liver biopsy will not be relegated to the dust bin.

The first edition of Dr. Scheuer's book was the first liver pathology text that I bought as a resident in 1968. I am thrilled that it has survived into its sixth edition, thus giving me the opportunity to recommend it to my residents. For those beyond the residency, let me add that this didactic masterpiece of the first order deserves to be read (and reread from time to time) not only by hepatopathologists but by generalists as well. In my opinion, it is still the best introductory text on liver biopsy interpretation. It is as good and authoritative a source of hepatopathological consultations as you will ever need in your daily practice.

\section{Ivan Damjanov \\ University of Kansas School of Medicine Kansas City, Kansas}

